\title{
O uso da música como estratégia de manejo comportamental em Odontopediatria
}

\author{
Music therapy as a behavioral management strategy in Pediatric Dentistry
}

Recebido: 20/10/2021 | Revisado: 25/10/2021 | Aceito: 26/10/2021 | Publicado: 31/10/2021

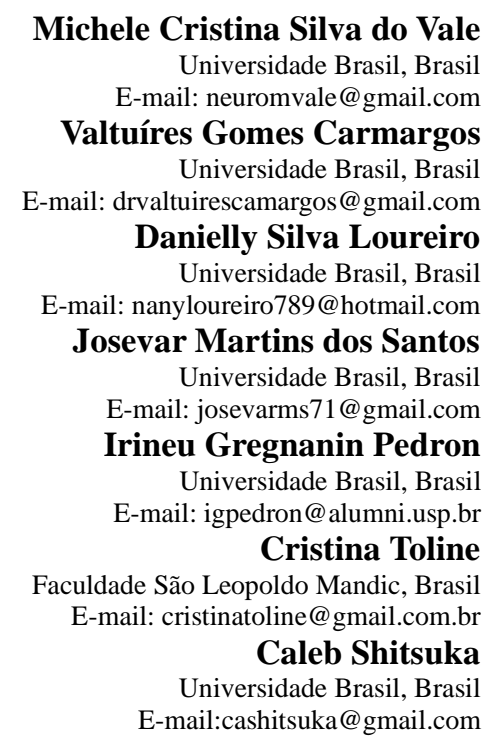

\begin{abstract}
Resumo
A relação comportamental do paciente infantil com o Odontopediatra tem sido abordada em muitos estudos devido ser potencialmente ansiogênica. Neste cenário, as técnicas de manejo comportamental surgem como valiosa ferramenta para estabilização e controle deste paciente. A musicoterapia se apresenta como uma alternativa terapêutica indolor para a criança. desta forma, este trabalho tem por objetivo realizar uma revisão de literatura, e apresentar a musicoterapia como ferramenta de manejo comportamental na odontopediatria. Esta revisão de literatura avaliou artigos publicados de 2008 a 2021, relacionados ao manejo comportamental em odontopediatria e o uso da musicoterapia como estratégia adjuvante ao tratamento convencional. Os artigos foram pesquisados nas bases de dados PubMed, Bireme, Scielo, Google Scholar e LILACS. Após avaliação, foram selecionados 29 artigos que constituem o corpo bibliográfico desta revisão. Conclusão: Concluiu-se que a música é uma alternativa não farmacológica eficaz para redução dos níveis de ansiedade em crianças frente o tratamento dentário.
\end{abstract}

Palavras-chave: Odontopediatria; Psicologia; Musicoterapia; Criança.

\begin{abstract}
The behavioral relationship between the child patient and the Pediatric Dentist has been addressed in many studies because it is potentially anxiogenic. In this scenario, behavioral management techniques emerge as valuable tools for stabilization and control of this patient. Music therapy presents itself as a painless therapeutic alternative for the child. Thus, this work aims to carry out a literature review and present music therapy as a behavioral management tool in pediatric dentistry. This literature review evaluated articles published from 2008 to 2021, related to behavioral management in pediatric dentistry and the use of music therapy as an adjuvant strategy to conventional treatment. Articles were searched in PubMed, Bireme, Scielo, Google Scholar and LILACS databases. After evaluation, 29 articles were selected that constitute the bibliographic body of this review. Conclusion: It was concluded that music is an effective non-pharmacological alternative to reduce anxiety levels in children when faced with dental treatment.
\end{abstract}

Keywords: Pediatric Dentistry; Psychology; Music therapy; Child.

\section{Introdução}

A odontopediatria é a especialidade odontológica que estuda as alterações, cuidados, e tratamentos do paciente infantil. Entretanto um dos maiores desafios para o Odontopediatra são as questões comportamentais que envolvem medo, e ansiedade, muito comuns nessa fase da vida. O comportamento não colaborativo, ou hostil do paciente pediátrico, tem muitas 
vezes relação direta com o insucesso da consulta, ou tratamento odontológico. Comportamentos de recusa, medo, ou fuga, podem influenciar de forma incisiva todas as etapas do tratamento desde anamnese, consulta, exames clínicos, exames laboratoriais, e terapia farmacológica. As fobias e, traumas psicológicos/familiares e sua relação com o atendimento odontológico, tem sido objeto de estudo na odontopediatria, com intuito de minimizar seus efeitos negativos permitindo o tratamento mais adequado para cada caso (Brandenburg \& Haydu, 2009; Possobom, 2007; Maltarollo et al., 2020; Moreira et al., 2021; Nogueira et al., 2021).

Destaca-se que a relação entre o profissional, e a criança, é descrita na literatura como potencialmente ansiogênica, para ambos, fator que pode impedir o início, ou continuidade do tratamento. Esse achado reforça a hipótese da necessidade de conhecimento e compreensão das ferramentas e técnicas de manejo comportamental em odontopediatria. Alguns estudos ainda sugerem que, os profissionais podem se sentir desmotivados, ou frustrados, frente a alguns comportamentos, e perfis de pacientes, e, tutores legais, desenvolvendo tolerância aos atendimentos destes. Brant, 2015; Tovo, et al., (2016), Vasconcellos et al., (2017) e Shitsuka et al, (2019), apontam que os responsáveis também podem influenciar a criança em relação ao medo, ansiedade e estresse, favorecendo um comportamento não-colaborativo.

$\mathrm{Na}$ busca de desenvolver novas ferramentas que mitiguem o comportamento de fuga ou recusa do paciente infantil, este tema tornou-se objeto de estudo, e discussão, o que tem gerado uma grande pesquisa acerca de protocolos, e técnicas, para driblar a ansiedade, medo, repulsa e o estresse. A psicologia refere que algumas situações podem gerar gatilhos de medo, ansiedade, evitação e fuga, comuns na infância, mudando apenas a percepção e entendimento do todo de acordo com a faixa etária. (Moreira, 2020). As técnicas de manejo comportamental apresentam-se como instrumento de grande importância para o entendimento, e controle, de comportamentos não-colaborativos. A comunicação é um dos fatores essenciais para uma boa relação entre o profissional e o paciente infantil, nessa esfera a psicologia agrega conhecimento a respeito das limitações, e características do desenvolvimento maturacional, e psicológico das crianças, contribuindo, de fato, para um melhor e mais eficaz atendimento clínico. (Brandenburg \& Haydu, 2009; Brandernburg \& Marinho-Casanova, 2013; Corrêa, 2013; Oliveira, 2014).

Para Anjos et al., (2017), Lisboa et al., (2018), Sousa et al., (2021) e, Tibola et al., (2021), o uso da música tem a capacidade de reduzir os níveis de estresse, ansiedade e nervosismo, com o intuito de modelar, e controlar comportamentos hostis que possam dificultar, ou impedir o atendimento. Dentre os benefícios citados, estão: redução de estresse, redução dos níveis de ansiedade, e aumento da aderência ao tratamento. Aliada a odontologia, essa estratégia consiste no uso de determinadas músicas que promovam relaxamento, diminuindo os níveis de ansiedade, e desconforto, com o intento de melhorar a relação interpessoal entre profissional, e paciente agregando uma experiência satisfatória, e não traumática ao atendimento

\section{Metodologia}

Esta revisão de literatura avaliou artigos publicados no período de 2008 a 2021, relacionados ao uso da musicoterapia como técnica de manejo comportamental em Odontopediatria e sua relação com o comportamento do paciente pediátrico. Foram selecionados artigos nas bases de dados como a Pubmed, Bireme, Scielo e LILACS. Os critérios de exclusão foram baseados na data de publicação e relação com o tema proposto pelos descritores. Por fim, selecionamos 29 artigos os quais constituem o corpo bibliográfico desta revisão.

\section{Discussão}

O medo, é uma emoção presente nos seres humanos desde a infância e se mantêm como parte presente do nosso cotidiano durante todo o desenvolvimento, perdurando por toda a vida. Entretanto, o medo pode ser benéfico quando bem 
direcionado, é uma forma de amadurecimento e enfretamento das questões que assolam o indivíduo. O medo frente a procedimentos odontológicos em pacientes pediátricos na maioria das vezes está associado a experiências prévias negativas da própria criança, ou, de seus pais, e tutores. (Andrade et al., 2020; Costa et al., 2020; Moraes et al., 2000).

Para Hass et al., 2016, Torres et al., 2020, o medo é uma emoção primária que apresenta diferentes manifestações de acordo com a faixa etária, e desenvolvimento maturacional da criança, quanto mais novas, mais expressivo é o nível de medo. Outro fator que parece ter relação com o medo em crianças, é a condição socioeconômica e experiências prévias da família, ou seja, os pacientes pediátricos com maior poder socioeconômico expressam mais medo quando apresentados a situações que envolvam atendimento odontológico, o mesmo para pacientes cujos pais ou tutores tenham relatado experiências odontológicas negativas, apresentam maior nível de medo e recusa.

Sensação de vulnerabilidade, aversão ao que lhe é desconhecido, e receio dos instrumentais podem estar associados ao comportamento não- cooperativo no paciente pediátrico. (Andrade et al., 2020).

Com o intuito de colaborar para um melhor, e mais adequado tratamento odontológico, as técnicas de manejo c tem por objetivo promover um comportamento mais cooperativo do paciente pediátrico frente ao profissional, e os procedimentos necessários. (Coelho et al., 2021).

Silva, e colaboradores (2016), sugerem que a técnica da distração é um método eficaz para desviar a atenção do paciente, por meio de músicas, desenhos, filmes, livros, e histórias, objetivando tirar o foco de um ato, ou procedimento que possa ser desagradável, ou traumático para a criança, diminuindo assim a percepção de desconforto. Dentro desta técnica existem dois métodos que podem ser trabalhados: a distração auditiva, e a distração visual.

A distração auditiva tem como instrumentos, músicas, áudios e histórias narradas partindo do uso de fones de ouvido. Já a técnica da distração visual, é baseada no uso de vídeos, óculos 3D, e histórias contadas em televisores (Rodrigues, 2020; Sant'anna, 2020).

A utilização da música para diminuição dos níveis de ansiedade e medo, têm sido frequente em muitas áreas da saúde, é sabido que a música parece atuar de forma fisiológica no organismo do indivíduo, reduzindo a pressão arterial. A música é apontada como uma ferramenta eficiente para a redução de medo e estresse no paciente pediátrico, além de ser uma alternativa não farmacológica para o declínio dos níveis de ansiedade, e cortisol. No âmbito emocional, a música otimiza os níveis do neurotransmissor serotonina, ativando as áreas cerebrais responsáveis pela recompensa, minimizando, em muitos casos, a percepção de estímulos dolorosos. O uso da música em ambiente odontológico tem se mostrado um instrumento paliativo eficaz, muitas vezes sendo até, de fato, alternativo a administração de sedativos, e ansiolíticos, aumentando a motivação e cooperação do paciente pediátrico. (Aravena et al, 2020; Tshiswaka et al, 2020).

Dixit, e colaboradores, (2020), investigaram a comparação da eficácia do uso de florais de Bach, e da musicoterapia no tratamento de pacientes pediátricos. O estudo contou com 120 crianças de quatro a seis anos de idade, alocados em três grupos, (grupo floral de bach, grupo musicoterapia, e grupo controle). Os pacientes dos três grupos foram submetidos ao procedimento de profilaxia oral, e aplicação tópica de flúor, e avaliados pela Escala de imagem facial, (FIS), parâmetros fisiológicos (pulsação) e escala de avaliação do comportamento. O uso da música como instrumento não farmacológico, para controle e diminuição parece ser significante quando relacionada a uma menor taxa de aumento da pressão arterial diastólica, pré-operatória quando comparadas ao grupo controle, e ao grupo florais de Bach. Entretanto, os resultados da escala facial de imagem não foram significantes quando comparados entres os três grupos. (Dixit et al, 2020).

Tshiswaka et al., (2020), apresentam uma avaliação do impacto que o uso da música desempenha durante o atendimento odontológico em pacientes pediátricos. A amostra foi comporta por 40 crianças com idade de cinco a onze anos, separados em dois grupos distintos, (grupo experimental, e grupo controle). Os pacientes foram avaliados por meio do exame de saturação de oxigênio, aferição da frequência cardíaca, instrumento de escala de ansiedade, e escala de dor de Corah. Os 
resultados apresentados se mostram favoráveis para o uso da musicoterapia na odontopediatria. Houve significativa diminuição da frequência cardíaca nos pacientes em que a música foi utilizada durante o tratamento. Todavia, os resultados se mostraram insignificantes na comparação dos dois grupos quando analisado saturação de oxigênio e escala de ansiedade e dor de Corah.

Brant, (2015), utilizou a música com objetivo de auxiliar a criança a passar pelo procedimento odontológico de maneira menos traumática. As análises foram realizadas por meio da aferição da frequência cardíaca, e saturação de oxigênio. $\mathrm{O}$ estudo contou com uma amostra de trinta e quatro crianças com idade entre quatro, e seis anos, sem experiência odontológica anterior. Os autores optaram pelo uso de música clássica e a escolhida foi a Sinfonia 40 em sol menor K550 de Wolfgang Amadeus Mozart. Os resultados demonstraram uma diminuição da saturação do oxigênio em um dos grupos durante a intervenção por meio da música, fortalecendo assim a tese sobre a eficácia da música como fator de relaxamento, e tranquilizante para atendimento do paciente pediátrico (Brant, 2015).

De acordo com Aravena et al., (2020), os resultados da musicoterapia são evidentes e significantes não apenas em crianças, ao realizarem uma comparação entre os efeitos do uso de músicas em $432 \mathrm{~Hz}$, e $440 \mathrm{~Hz}$, frente a ansiedade, e os níveis de cortisol salivar durante extrações dentárias. Foram incluídas no estudo quarenta e duas mulheres, com média de idade de 23,8 anos, diagnosticadas com ansiedade moderada, e dividas em três grupos, (grupos $432 \mathrm{~Hz}$ e grupo $440 \mathrm{~Hz}$ e grupo sem música). Observou-se que o nível de ansiedade foi significativamente mais baixo no grupo submetido a músicas em $432 \mathrm{~Hz}$ quando comparadas ao grupo controle. A análise de cortisol foi significativamente menor em $440 \mathrm{~Hz}$, na comparação como grupo controle. Desta forma os resultados sugerem que o uso da música em $432 \mathrm{~Hz}$ foi mais eficaz para a diminuição dos níveis de ansiedade, e níveis de cortisol salivar, quando comparado ao grupo controle, reforçando a tese de que a música é benéfica durante o atendimento odontológico. (Aravena, et al., 2020).

A musicoterapia é uma técnica que pode ser ensinada tanto para alunos de graduação quanto para alunos de pósgraduação, para que sejam aplicadas à prática profissional. Cabe ao cirurgião dentista odontopediatra obter o domínio da técnica e assim conduzir o atendimento de modo mais acolhedor, utilizando essa técnica de manejo comportamental para colaboração do paciente infantil. (Valente et al., 2021; Santo, 2016, e Rocha et al., 2021). Desta forma, o profissional obtém o controle do estresse, da ansiedade, e do medo da criança proporcionando assim um atendimento odontológico mais familiar, acolhedor e menos traumático.

\section{Conclusão}

Conclui-se que a musicoterapia como estratégia de manejo comportamental em pacientes pediátricos é eficaz, pois produz considerável redução dos níveis de estresse, ansiedade, e medo, frente a consulta odontológica. Baseando-se em troca de experiências, e sensação de relaxamento provocada pelos acordes musicais. Entretanto, assim como outras técnicas e estratégias de manejo comportamental, é evidente que a musicoterapia possui indicação, e cabe ao profissional por meio de estudos, especializações, e cursos, estabelecer a técnica de manejo mais adequada para ser usada isoladamente, ou associada as demais técnicas, a fim de obter o melhor tratamento para o paciente pediátrico.

\section{Agradecimentos}

A senhora Rute por todo apoio e incentivo. Aos professores do curso de Odontologia da Universidade Brasil, campus Itaquera. Ao professor Hatsuo Kubo (in memorian).

\section{Referências}

Anjos, A. G., Montanhaur, C. D., Campos E. B. V., Piovezana, A. L. P. D., Montalvão, J. S., \& Neme, C. M. B., (2017). Musicoterapia como estratégia de intervenção psicológica em crianças: uma revisão de literatura. Gerais: Revista Interinstitucional de psicologia. 10(2), 228-238. 
Aravena, P. C., Almonacid, C., \& Mancilla, M. I. (2020). Effect of music at $432 \mathrm{~Hz}$ and $440 \mathrm{~Hz}$ on dental anxiety and salivary cortisol levels in patients undergoing tooth extraction: a randomized clinical trial. Journal of Applied Oral Science. 28 [Accessed 6 October 2021] , e20190601. Available from: <https://doi.org/10.1590/1678-7757-2019-0601>.

Brandenburg, O. J., \& Haydu, V. B., (2009). Contribuições da análise do comportamento em odontopediatria. Revista de psicologia ciência e profissão, 29(3), $462-477$.

Brandenburg, O, J., \& Marinho-Casanova, M. L., (2013). A relação mãe-criança durante o atendimento odontológico: Contribuições da análise do comportamento. Estudos de psicologia, 30(4), 629-640.

Brant, M. O. (2015). A música como estratégia de distração durante o atendimento odontológico de crianças um ensaio clínico cruzado. Programa de pós graduação UFMG.

Cardoso, C. L., \& Loureiro, S. R. (2008) Estresse e comportamento de colaboração em face do tratamento odontopediátrico. Revista de psicologia em Estudo, 13(1), 133-141.

Coelho, V. F. D., Coelho, L. V. D., \& Costa, A. M. G. (2021). Técnicas de manejo em Odontopediatria: uma revisão narrativa da literatura. Research, Society and Development, 10(11), e414101119489. https://doi.org/10.33448/rsd-v10i11.19489

Costa, I. 1. C., Moura, K. R. B., Casimiro, L. S., Medeiros, R. M. L., Filho, J. M. C. V., \& Vieira, A. P. S. B. (2020). Medo infantil frente ao tratamento odontológico: Uma revisão de literatura. Revista diálogos em saúde. 3(2), 25-37

Corrêa, M. S. N. P., (2013). Conduta Clínica e Psicológica na Odontopediatria, 1(2), 620.

Dixit, U. B., \& Jasani, R. R. (2020). Comparison of the effectiveness of Bach flower therapy and music therapy on dental anxiety in pediatric patients: A randomized controlled study. Journal of the Indian Society of Pedodontics and Preventive Dentistry, 38(1), 71-78. https://doi.org/10.4103/JISPPD.JISPPD_229_19

Hass, M. G. M., Oliveira, L. J. C., \& Azevedo, M. S. Influência da vestimenta do cirurgião-dentista e do ambiente do consultório odontológico na ansiedade de crianças préescolares durante consulta odontológica: resultados de um estudo piloto. RFO. 21(2), 201-207.

Maltarollo, T. H., Pedron, I. G., Medeiros, J. M. F., Kubo, H., Martins, J. L., \& Shitsuka, C. (2020). A erosão dentária é um problema! Research, Society and Development, 9(3), e168932723. https://doi.org/10.33448/rsd-v9i3.2723.

Moraes, A. B. A., Bovi Ambrosano, G. M., \& Singh, K. A. Medo, ansiedade e controle relacionados ao tratamento odontológico. Pesq Odont Bras. 14(2), 131136.

Moreira, J. S.., Vale, M. C. S. do., Francisco Filho, M. L., Souza, K. M. N. de, Santos, S. C. C. dos, Pedron, I. G, \& Shitsuka, C. (2021). Técnicas de manejo comportamental utilizados em odontopediatria frente ao medo e ansiedade. E-Acadêmica, 2(3), e032334. https://doi.org/10.52076/eacad-v2i3.34

Nogueira, E. C. P., Bussadori, S. K., Santos, E. M., Imparato, J. C. P., \& Rezende, K. M. (2021). O uso do Papacárie® como estratégia do controle do estresse na odontopediatria. Research, Society and Development, 10(12), e491101220810. https://doi.org/10.33448/rsd-v10i12.20810

Oliveira, C. C. O. (2014). Atividades lúdicas na odontopediatria: Uma breve revisão literaria. Revista Brasileira de Odontologia. 71(1), 103-7.

Possobom R. F., Carrascoza, K. C., Moraes A. B. A. \& Costa Jr, A. L. (2007). O tratamento odontológico como gerador de ansiedade. Psicologia em Estudo. $12(3), 609-616$

Rocha S. S. D., Joye C. R., \& Moreira, M. M. (2020) A Educação a Distância na era digital: tipologia, variações, uso e possibilidades da educação online. RSD. 9(6):e10963390. https://rsdjournal.org/index.php/rsd/article/view/3390

Rodrigues, M. A. C., \& Rocha, N. M. (2020). Técnica da distração audiovisual para controlar a ansiedade em crianças no tratamento odontológico. Brazilian Journal of Implantology and Health Sciences. 2(9), 26-36.

Sant'anna, R. M, Silva, R. A., Silva, L. V., \& Almeida, T. F. (2020). Aspectos éticos e legais das técnicas de manejo de comportamento em odontopediatria: uma revisão narrativa da literatura. Rev Bras Odontol LegRBOL. 7(2), 70-80.

Santo E do E. (2016) Ensinar e aprender na Educação a Distância: um estudo exploratório na perspectiva das práticas tutoriais. RSD [Internet]. $8^{\circ}$ de dezembro de 2016, 3(2):92-114. https://rsdjournal.org/index.php/rsd/article/view/16

Silva, L. F. P., Freire, N. C., Santana, R. S. \& Miasato, J. M. (2016). Técnicas De Manejo Comportamentais não farmacológicas na odontopediatria. Rev. Odontol. 135-42.

Shitsuka, C., Friggi, M. N. P., \& Volpini, R. M. C. (2019). Influência dos pais sobre o comportamento infantil no atendimento odontológico. Research, Society and Development, $8(7)$, e43871154. https://doi.org/10.33448/rsd-v8i7.1154

Sousa, A. N. da S., Saraiva, M. S., Machado, T. V. R., \& Souza, J. C. P. de. (2021). A utilização da musicoterapia no tratamento de idosos diagnosticados com a doença de Alzheimer. Research, Society and Development, 10(12), e112101220010. https://doi.org/10.33448/rsd-v10i12.20010

Valente, G. S. C., Moraes Érica, B. de, Sanchez, M. C. O., Souza, D. F. de, \& Pacheco, M. C. M. D. (2020) O ensino remoto frente às exigências do contexto de pandemia: Reflexões sobre a prática docente. $R S D ; 9(9):$ 843998153. https://rsdjournal.org/index.php/rsd/article/view/8153

Vasconcellos, C., Imparato, J. C. P., \& Rezende K. M. (2017). Motivation chart as supporting tool in pediatric dentristry. RGO. Revista Gaucha de Odontologia. 65(3), 276-281. 
Tibola, C.., et al. (2021). Recursos não-farmacológicos para alívio da dor no trabalho de parto: Relato de experiência e revisão integrativa. Research, Society and Development, 10(7), e18310716446. https://doi.org/10.33448/rsd-v10i7.16446

Tshiswaka, S. K., \& Pinheiro, S. L. (2020). Efeito da música na redução da ansiedade em crianças durante o tratamento odontológico. RGO - Revista Gaúcha de Odontologia 68, e20200033. https://doi.org/10.1590/1981-863720200003320190049.

Torres, M. E. B. B., Souza, K. L. B., \& Cruz, V. S. A. (2020). Estratégias de controle do medo e ansiedade em pacientes odontopediátricos: revisão de literatura. Revista Eletrônica Acervo Saúde, 12(11), e5213. https://doi.org/10.25248/reas.e5213.2020

Tovo, M. F., Faccin, E. S., \& Vivian, A. G (2016). Psicologia e odontopediatria: Contextualização da interdisciplinaridade no Brasil. Aletheia, 49(2) ,76-88. 